Artículo

\title{
Análisis bibliométrico de la producción científica sobre cadena de valor turística
}

\author{
María Isabel Rosas Jaco ${ }^{1}$ \\ Silvia Xochilt Almeraya Quintero ${ }^{1 \S}$ \\ Lenin Gerardo Guajardo Hernández ${ }^{1}$ \\ Dora Ma. Sangerman-Jarquín ${ }^{2}$
}

${ }^{1}$ Colegio de Postgraduados-Campus Montecillo. Carretera México-Texcoco km 36.5, Montecillo, Texcoco, Estado de México. CP. 56230. (maria.rosas@colpos.mx; xalmeraya@colpos.mx; glenin@colpos.mx). ${ }^{2}$ Campo Experimental Valle de México-INIFAP. Carretera Los Reyes-Texcoco km 13.5, Coatlinchan, Texcoco, Estado de México, México. CP. 56250. Tel. 55 38718700, ext. 85353. (sangerman.dora@inifap.gob.mx).

${ }^{\S}$ Autora para correspondencia: xalmeraya@colpos.mx.

\section{Resumen}

Para desarrollar el potencial turístico de una comunidad local, región o destino turístico en general es importante identificar cada uno de los elementos que lo componen y como es que éstos trabajan. Esta información es recabada y publicada, en su mayoría, en artículos científicos. Por lo que el objetivo central de esta investigación es analizar el estado del conocimiento, mediante un estudio bibliométrico retrospectivo, la producción científica que desarrolle mencione y proponga elementos relacionados con el tema cadenas de valor con enfoque turístico destacando factores como autor, afiliación, país, tipo de documento, entre otros. La metodología de la investigación se basa en una revisión documental e información bibliométrica retrospectiva de artículos relacionados con el tema de cadenas de valor turísticas. Para ello, se consultó la base de datos Scopus haciendo la búsqueda del tema 'tourism value chain' escrita en el título, resumen o palabras clave del artículo, teniendo como resultado 52 trabajos de investigación realizados entre los años 2010-2020. La consulta de dichos documentos se realizó entre el 20 de julio 2020 al 25 de junio de 2021 en el sitio web www.scopus.com. Se concluye que los estudios sobre cadenas de valor turísticas de los últimos diez años son pocas, en donde predomina la modalidad de artículo y como país líder Tailandia, aunque también hay países Latinoamericanos que pueden sentar las bases de este tema y visibilizar la importancia de la investigación de cadenas de valor turísticas para lograr un desarrollo equitativo entre todos los eslabones de la cadena.

Palabras clave: cadenas de valor, calidad, indicadores bibliométricos, servicios, turismo.

Recibido: mayo de 2021

Aceptado: julio de 2021 


\section{Introducción}

El énfasis que se le da en muchos casos a los bienes tangibles lleva a ignorar a los servicios, los cuales llegan a representar el sector más grande y dinámico de muchas naciones. En las sociedades desarrolladas pueden ocupar a más de $60 \%$ de la población, mientras que en países más pobres la población que se dedica a este sector, especialmente al pequeño comercio, oscila entre 10 y $40 \%$. Tomando como referencia a México se tiene que, de todos los sectores de actividad económica, el sector servicios como el turismo (21.5\%), la transformación (18.2\%) y el comercio (17.6\%) emplean el mayor porcentaje de jóvenes entre 16 y 24 años (Observatorio laboral, 2020).

Las economías de servicio no se limitan a prevalecer en los países desarrollados, en los países en vías de desarrollo, la mayoría de la población activa está a menudo empleada en actividades tan variadas como la educación, el comercio, la administración, las finanzas, la seguridad privada, el transporte y el turismo, entre otros (Kotler et al., 2011). El sector terciario, el cual se dedica a ofrecer servicios necesarios para el funcionamiento de la economía, es el más heterogéneo de toda la actividad económica, ya que no produce bienes, sino servicios (Serrano, 2011), lo que lo hace un sector muy dinámico y en constante cambio.

Autores como Romero y Tejada (2011); Kaplinksy y Morris (2001) hacen referencia a que las actividades turísticas forman parte del sector terciario y están conformadas por diferentes actores, los cuales se pueden clasificar en cuatro niveles: planificadores o diseñadores del producto turístico básico, proveedores de productos y servicios, intermediarios turísticos y los propios turistas. Estos se vinculan entre sí para satisfacer la demanda en los destinos, por lo que la cadena de valor con enfoque turístico (CVT) es una forma de organizar al sector turístico identificando los eslabones que lo integran, cuáles son sus interrelaciones para mejorar procesos de comunicación y alcanzar beneficios más equitativos entre todos los implicados directa e indirectamente en esta actividad; asimismo, identifica puntos críticos y restricciones que deben atenderse.

De acuerdo con la Organización Mundial del Turismo (2015), dicho sector es la clave para el desarrollo, la prosperidad y el bienestar de un país, por lo que con los datos extraídos a partir de la base de datos Scopus, se realiza un análisis del estado del conocimiento, mediante un estudio bibliométrico retrospectivo, de la producción científica que desarrolle, mencione y proponga elementos relacionados con el tema CVT destacando factores como autor(a), afiliación, país, tipo de documento, entre otros. Los resultados de dicho análisis permitirán visualizar la importancia que tiene este tema tanto para los investigadores como para los actores locales involucrados en la toma de decisiones, así como identificar las investigaciones que han tenido mayor impacto entre la comunidad científica al formar parte de una estrategia de desarrollo integral.

\section{Materiales y métodos}

La investigación se basa en una revisión documental e información bibliométrica retrospectiva de artículos relacionados con el tema de cadenas de valor turísticas. Para ello, se consultó la base de datos Scopus haciendo la búsqueda del tema 'Tourism Value Chain' escrita en el título, resumen o palabras clave del artículo, teniendo como resultado 52 trabajos de investigación realizados entre los años 2010-2020. La consulta de dichos documentos se realizó entre el 20 de julio 2020 al 25 de junio de 2021 en el sitio web www.scopus.com. 


\section{Resultados y discusión}

El turismo forma parte del sector servicios, por lo que la cultura de servicio se centra en atender y satisfacer las demandas del cliente o turista. Sin mencionar una empresa en específico, la cultura del servicio y la calidad de este debe iniciar desde la gerencia o puesto más alto y continuar con el resto de los empleados que laboran en la empresa, así como de toda la cadena productiva que interviene para la prestación del servicio.

La calidad en el servicio es un tema muy importante en turismo, no solo en los productos o servicios terminados, sino en la calidad de los procesos y personas que intervienen en dichos productos o servicios. La calidad pasa por todas las fases de la actividad de la empresa; es decir, por todos los procesos de diseño, producción, venta y mantenimiento de los productos o servicios (Imai, 1998). Con respecto al valor que se le da al servicio, este dependerá si el consumidor reconoce la importancia del beneficio ofrecido (González, 2015). Sin embargo, la investigación de este tema se ha centrado básicamente en calidad y satisfacción, el valor que se le da al servicio y a todos los elementos que intervienen en él no han tenido suficiente nivel de estudio hasta el momento (Duque, 2005).

Es necesario puntualizar cuáles son las características de los servicios. Al respecto, Kotler (1997) menciona que un servicio es 'cualquier actividad o beneficio que una parte ofrece a otra y que es intangible y no tiene como resultado la posesión de algo'. Por lo que el turismo tiene una relación inherente con la prestación de servicios. Los turistas lo único que se llevan del lugar visitado y prevalece por muchos años es la experiencia vivida que les permite crear recuerdos memorables.

\section{Entre las características de los servicios se encuentran}

\section{Intangibilidad}

A diferencia de los productos físicos, los servicios no se pueden experimentar a través de los sentidos antes de ser consumidos. Robert et al. (1989) menciona que cualquiera que compra un servicio se puede ir con las manos vacías, pero nunca se irá con la cabeza vacía, haciendo referencia a la obtención de experiencias a través de la prestación de un servicio.

\section{Inseparabilidad}

Tanto el prestador de servicios como el cliente o turista debe estar presente para que el servicio sea brindado. El carácter indisociable del servicio significa que los turistas forman parte indispensable del producto.

\section{Variabilidad}

La calidad del servicio depende en todo momento de quién, cuándo, cómo y dónde se suministre el servicio. En este sentido, la falta de homogeneidad en el producto puede ser la causa principal de decepción en los turistas, o por el contrario, puede ayudar a ofrecer servicios a la medida de cada visitante. 


\section{Carácter perecedero}

Los servicios no se almacenan ni se guardan para ser utilizados posteriormente, se extinguen con su prestación, por lo que no se pueden almacenar las existencias no vendidas.

Los servicios turísticos ofrecidos por las agencias de viajes, hoteles, restaurantes, guías de turistas, etc., no pueden ser vistos o tocados por los turistas, a pesar de ver o tocar algunos de los elementos tangibles del producto turístico (Zeithaml et al., 2009). Estos son producidos y consumidos en el momento, tanto por los prestadores de servicios como del mismo turista, por lo tanto, la satisfacción del cliente o turista está determinada por la calidad que tengan los servicios adquiridos a lo largo de su estancia en el destino.

Como se mencionó anteriormente, la calidad del servicio al turista es la clave para lograr éxito dentro de la competencia que exige este mundo globalizado. Los turistas son personas más informadas y con estudios superiores que demandan calidad en los servicios ofertados, por lo tanto, la fidelidad del cliente es tal vez, la mejor forma de medir la calidad. La habilidad que tenga una empresa de servicios para captar y fidelizar a los clientes dependerá en gran medida del valor añadido que esta les ofrezca (Kotler, 2011).

Ahora bien, la calidad en el servicio está estrechamente ligada con la cadena de valor, misma que funciona como una herramienta innovadora y útil para identificar los factores que impulsen o condicionan la generación de valor en un destino turístico y en cada uno de sus eslabones (Pulido y López, 2016). Por lo anterior, no se debe olvidar que detrás de cada servicio turístico está un sistema tecnológico que lo respalda, además de contar con una cadena de valor que está integrada por proveedores hasta llegar al cliente final (Izaguirre, 2015).

En lo que respecta al concepto de cadena de valor, éste fue creado y difundido por Porter y Millar, (1985) el cual definió valor como la cantidad que el comprador está dispuesto a pagar por un producto que ofrece la empresa. Las actividades de valor para una compañía según Porter se conforman por nueve actividades, las primeras cinco son primarias las cuales envuelven la creación física del producto (logística interna, operaciones, logística externa, marketing, ventas y servicios) y cuatro actividades de apoyo que sustentan a las primarias (infraestructura de la empresa, gestión de recurso humano, desarrollo tecnológico y aprovisionamiento) (Porter y Millar, 1985).

Al respecto, Alonso (2008) menciona que la cadena de valor que propone Porter, a pesar de enfocarse directamente a aspectos tangibles puede ser adaptada a la prestación de servicios. Siguiendo con algunas otras definiciones, se denomina cadena de valor al conjunto de actividades principales de una empresa, que se unen a través de eslabones, a medida que el producto pasa por cada una de estas actividades va añadiendo su valor (Kippenberger, 1997).

Bajo esta misma perspectiva, una cadena de valor simple describe la gama completa de actividades que se requieren para llevar un producto o servicio desde su concepción, a través de las diferentes fases de producción (que implican una combinación de transformación física y el aporte de varios servicios de productor), entrega a consumidores finales y final eliminación después del uso. (Kaplinsky y Morris, 2001). La cantidad de eslabones de una cadena de valor depende del tipo de industria. Éstas pueden llevarse a cabo en ocasiones por una empresa y en otras, por varias (Kaplinsky, 2000). 
Para la Organización de las Naciones Unidas para la Alimentación y la Agricultura (FAO) (2006) una cadena de valor es como una red o alianza estratégica que se establece, formal o informalmente, entre un número de actores empresariales independientes que participan dentro de una o más cadenas productivas. Para la Agencia Alemana de Cooperación Técnica (GTZ) (2007), una cadena de valor es definida como: 'un sistema económico compuesto por los operadores de este, prestadores de servicios operacionales y sus vínculos comerciales a nivel micro, y por los prestadores de servicios de apoyo a nivel meso. Se consideran parte de la cadena de valor a todos los operadores que agregan valor a un determinado producto mercadeable, comenzando por la materia prima hasta llegar al consumidor final'.

Este enfoque metodológico de ValueLinks creado por la Agencia Alemana busca el desarrollo económico sectorial a nivel local, nacional y global, a través del fomento de cadenas de valor en beneficio de la población con menores recursos económicos, con enfoque en el crecimiento propobre (CPP). Este enfoque tiene premisa de que el crecimiento económico de la gente pobre en los mercados brinda una solución sostenible al problema de la pobreza (GTZ, 2007).

En el manual ValueLinks, de la agencia alemana de cooperación internacional (GTZ por sus siglas en alemán) hace una distinción de cuatro actores que intervienen en las cadenas de valor: en primera instancia están los operadores de la cadena de valor conformado por el sector privado como protagonistas de la cadena, en segundo lugar, están los prestadores de servicios y apoyo como pueden ser las cámaras e institutos tecnológicos, organismos del sector académico o universidades. Como tercer elemento está la administración pública para crear condiciones favorables para el desarrollo económico, y finalmente están las agencias de desarrollo y ONG para impulsar, fomentar y apoyar al resto de los agentes de la cadena de valor (GTZ, 2007).

Por lo anterior, la comprensión de las cadenas de valor con enfoque en turismo permite organizar al sector turístico en el cual intervienen los proveedores de todos los productos y servicios que forman parte de la experiencia que vivirá el turista, desde la planificación del viaje, la permanencia en el destino y selección de servicios, hasta llegar a su lugar de residencia. Para los investigadores y profesionales del sector, ayuda a identificar aquellas cadenas de valor en las que los actores locales, nacionales y globales pueden producir mejores productos y servicios, aumentar la creación de empleos y en muchos casos, reducir la pobreza que se vive en la mayoría de los países en vías de desarrollo. Finalmente, la investigación y posterior implementación de este tema es de vital importancia para generar cambios tangibles y mejorar la distribución de los ingresos y beneficios en las comunidades que participan en la actividad turística.

Una vez realizada la búsqueda de artículos científicos se analizaron diferentes aspectos de los mismos, los cuáles se detallan a continuación: el interés por el tema de cadenas de valor enfocadas a servicios turísticos lo muestra Ortíz (2001) al publicar su investigación Estia-efficient electronic services for tourists in action, por lo que a partir de ese año los escritos han ido en aumento paulatinamente. Tomando en cuenta los 10 años retrospectivos para este análisis se tiene (Figura 1) que los picos más altos en publicaciones se dieron en 2019 y 2020 con siete y nueve documentos respectivamente. La emergencia sanitaria por el virus COVID-19 ha golpeado duramente al sector turístico, por lo que se ha vuelto una necesidad hacer investigación sobre cómo mejorar y adaptar la actividad turística a la 'nueva normalidad'. 


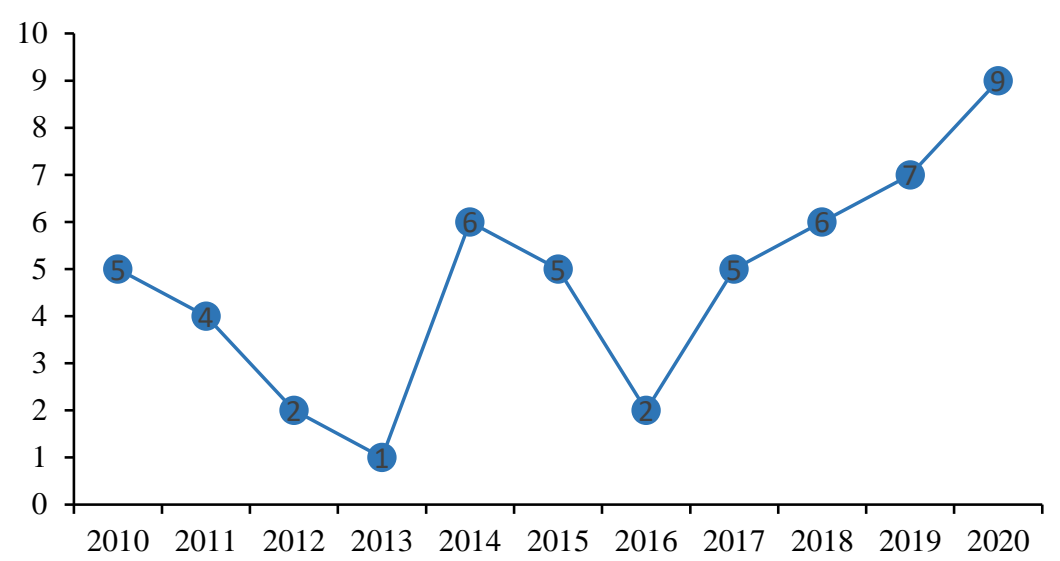

Figura 1. Número de documentos escritos por año (2010-2020). Elaboración con datos de www.scopus.com.

Los autores que tienen publicaciones sobre temas relacionados con CVT se encuentran desglosados en el Figura 2. En este se puede apreciar un listado de 12 autores(as) que tienen dos trabajos de investigación, algunas como único autor y otras en colaboración con otros investigadores. Por ejemplo: Adiyia y Vanneste (2015 y 2018) tienen trabajos en conjunto publicados. Es el mismo caso para Casarin et al. (2014) al tener dos trabajos colaborativos. Halpern y Mwesiumo (2016 y 2018) colaboraron en dos artículos. Okolo-Obasi y Uduji (2019 y 2020) trabajaron en colaboración con otros autores y finalmente Anderson (2017 y 2018) tiene una investigación cómo único autor en 2018 y otra en colaboración en 2017. El único que tiene dos contribuciones publicadas como único autor es Kaewkitipong (2010a) y (2010b), aunque el título de la investigación sea el mismo.

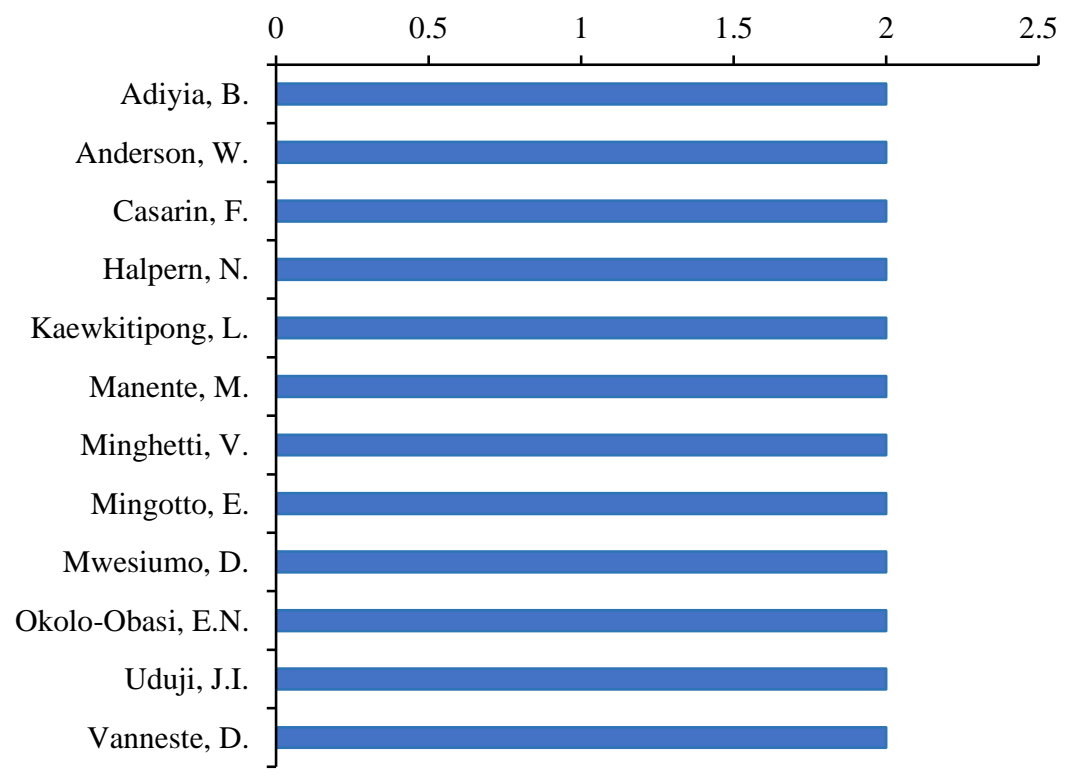

Figura 2. Autores(as) de artículos sobre cadenas de valor turísticas. Elaboración con datos de www.scopus.com. 
Conocer la afiliación de los trabajos de investigación publicados es importante, ya que permite identificar la institución educativa a la que pertenecen los investigadores, mismos que podrían ser opción para futuras colaboraciones con respecto a temas de CVT. Al respecto en la (Figura 3), se muestra la afiliación de los investigadores. En primer lugar se encuentra la Universidad de Innsbruck, una de las más antiguas de Austria, seguida de la Universidad Thammasat, segunda universidad más antigua de Bangkok en Tailandia. En tercer lugar se encuentra la Universidad Ca'Foscari, principal universidad de Venecia en Italia.

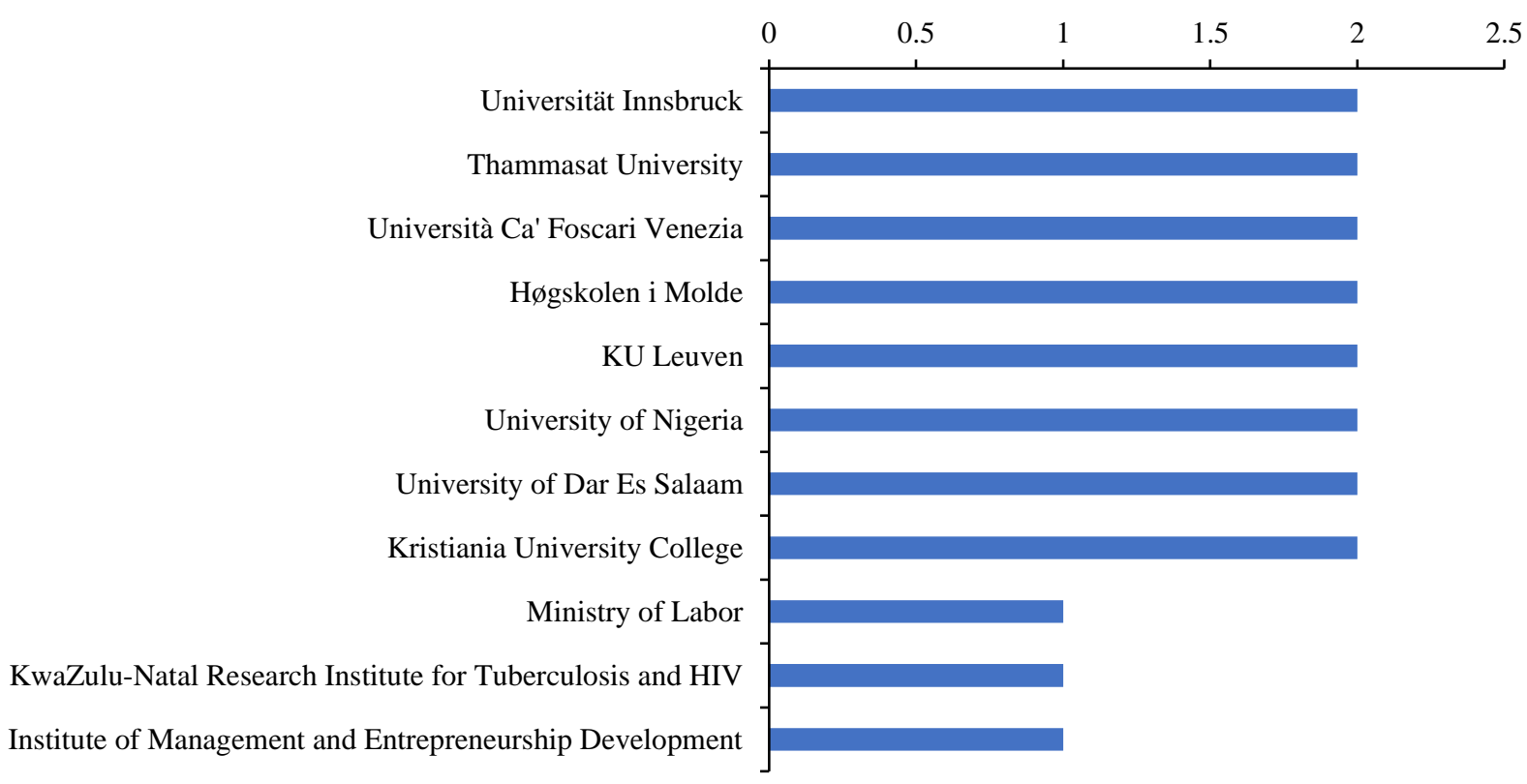

\section{Figura 3. Documentos por afiliación. Elaboración con datos de www.scopus.com.}

En el listado están dos universidades de Noruega, una de ellas es la Universidad de Molde (Høgskolen i Molde) y la otra es la Kristiania University College, una de las instituciones privadas de educación superior más antiguas ubicada en Oslo. Finalmente están la KU Leuven, universidad más grande y mejor posicionada de Bélgica, líder en investigación y co fundadora de la Liga de Universidades Europeas de Investigación (LERU) y la Universidad de Nigeria en Nsukka, Nigeria.

El país lider en investigaciones cientificas que retoman temas relacionados con cadenas de valor turísticas es Tailandia al tener el mayor número de documentos escritos (dos en 2010, uno en 2015 y recientemente publicó dos en 2020), le sigue China con cuatro investigaciones (uno en 2013, dos en 2014 y uno en 2019) al igual que Sudáfrica (tres en 2018 y uno en 2019). Dinamarca cuenta con tres escritos, igual que Italia, Noruega y Estados Unidos de América publicados en diferentes años (Figura 4).

Cabe destacar, en esta sección que de las nueve contribuciones realizadas este año 2020 con temas relacionados a CVT estos pertenecen a países como Colombia, Alemania, Corea del Sur, España, Suiza, Italia, Nigeria y nuevamente los países que se encuentra entre los primeros diez en publicaciones son India y Tailandia. 


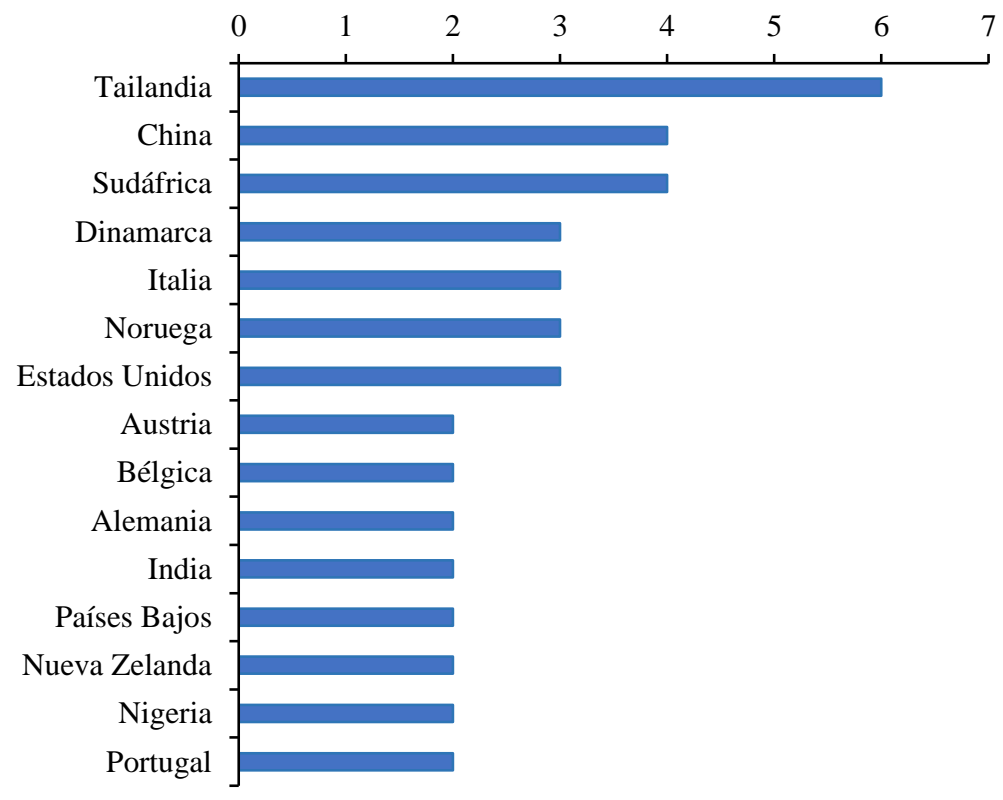

Figura 4. Países con mayor número de investigaciones en CVT. Elaborado con datos de www.scopus.com.

Como mención especial, se reconoce la contribución que han realizado los países Latinoamericanos (Figura 5), ya que a pesar de ser mínima su producción científica con relación al tema CVT sirven de referencia para futuras investigaciones dentro del contexto latinoamericano. ¿Uno de ellos es el realizado en Ecuador por Rendón y Bidwell (2015) el cual es un capítulo de libro titulado 'Success in progress? tourism as a tool for inclusive development in Peru's Colca Valley', con afiliación en la Facultad Latinoamericana de ciencias sociales en Quito y de la Victoria University of Wellington, Wellington, Nueva Zelanda.

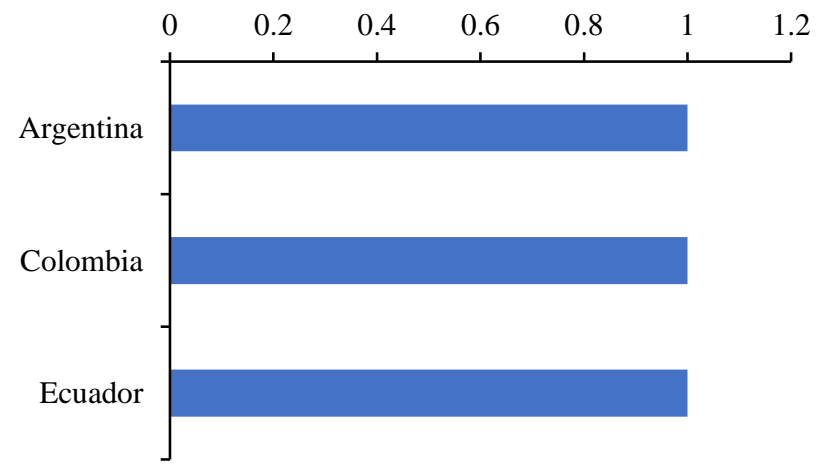

Figura 5. Países latinoamericanos con investigaciones en CVT. Elaboración con datos de www.scopus.com.

Le sigue Argentina con el artículo de Castillo et al. (2017) título: 'The causal effects of regional industrial policies on employment: a synthetic control approach'. Las instituciones de afiliación son el observatorio de empleo y dinámica empresarial, ministerio de trabajo, Universidad de San Andrés y Corporación Interamericana de Inversiones. 
Finalmente, una contribución reciente de Colombia es la realizada por González y Husain (2020) con el trabajo escrito titulado 'Social entrepreneurship and sustainable tourism in Colombia: a baseline study in post-conflict regions'. Afiliación con la Universidad de la Salle y Corporación Universitaria Minuto de Dios, ambas en Bogotá Colombia.

Con respecto al tipo de documentos escritos relacionados a CVT se tiene un registro de 36 artículos científicos $(71 \%)$, seguido de siete capítulos de libro (13\%), cinco documentos de conferencias $(10 \%)$ y finalmente con un libro, una nota y un documento en revisión con $2 \%$ respectivamente (Figura 6).

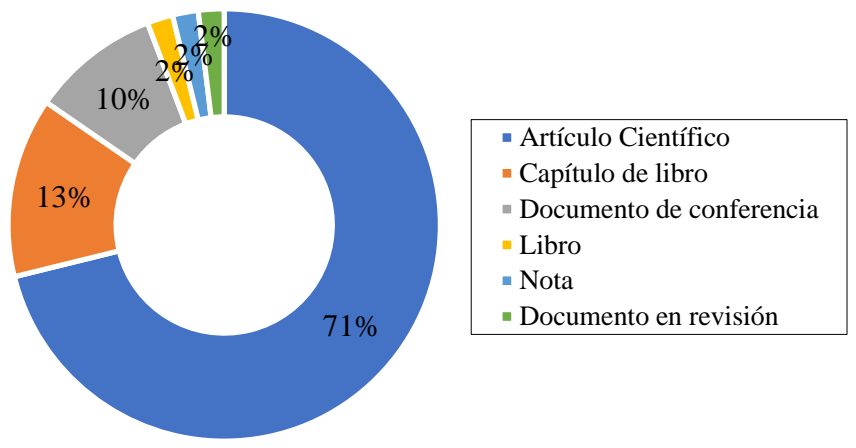

Figura 6. Tipo de publicaciones relacionadas con CVT. Elaboración con datos de www.scopus.com.

La temática de los escritos permite conocer cuál es el área que pertenecen los investigadores interesados en desarrollar temas relacionados con CVT (Figura 7). Al respecto, se muestra que el área de negocios, gestión y contabilidad tiene $41 \%$, lo que equivale a 43 escritos. Las ciencias sociales registraron $30 \%$, lo que se traduce a 31 escritos. Es importante destacar, esta área del conocimiento ya que a ella pertenece el turismo y en la cual se han formulado diversidad de investigaciones. Economía, econometría y finanzas es otra de las áreas interesadas en el tema de CVT la cual examinó 8\%, resultando en nueve documentos. Un área, que se ha desarrollado en esta temática son las ciencias medioambientales, que busca que las actividades turísticas se desarrollen basadas en los principios de la sustentabilidad, por lo que observó $7 \%$ con siete documentos. Finalmente, ciencias de la computación, artes y humanidades, así como la ingeniería, ciencias exactas, energía y matemáticas registran un interés en el tema, aunque en menor porcentaje.

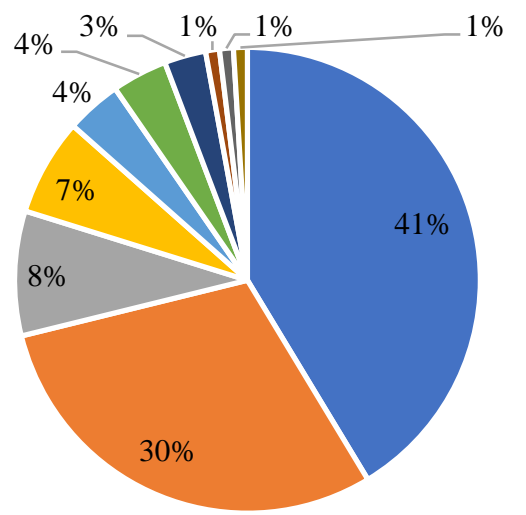

- Negocios, Gestión y Contabilidad

- Ciencias Sociales

- Economía, Econometría y Finanzas

- Ciencias Medioambientales

- Ciencias de la Computación

- Ingeniería

- Artes y Humanidades

- Ciencias exactas

- Energía

- Matemáticas

Figura 7. Documentos por área temática sobre CVT. Elaboración con datos de www.scopus.com. 
Para concluir con el análisis de gráficas se encuentran las revistas más solicitadas para publicar temas relacionados con CVT. Al respecto se muestra que la revista African Journal Of Hospitality, Tourism and Leisure es la más popular al tener seis documentos entre el 2018 y 2020, le sigue Tourism Review con un documento en 2016 y dos en 2017. Development Southern Africa con dos documentos (2010 y 2018). La revista Journal of Tourism and Cultural Change aunque sólo tiene dos publicaciones ambas son actuales ya que pertenecen al año 2019 y 2020. Tourism Planning and Development y Wit Transaction on Information and Communication Technologies cuentan con dos escritos cada una publicados entre 2010 y 2018 (Figura 8).

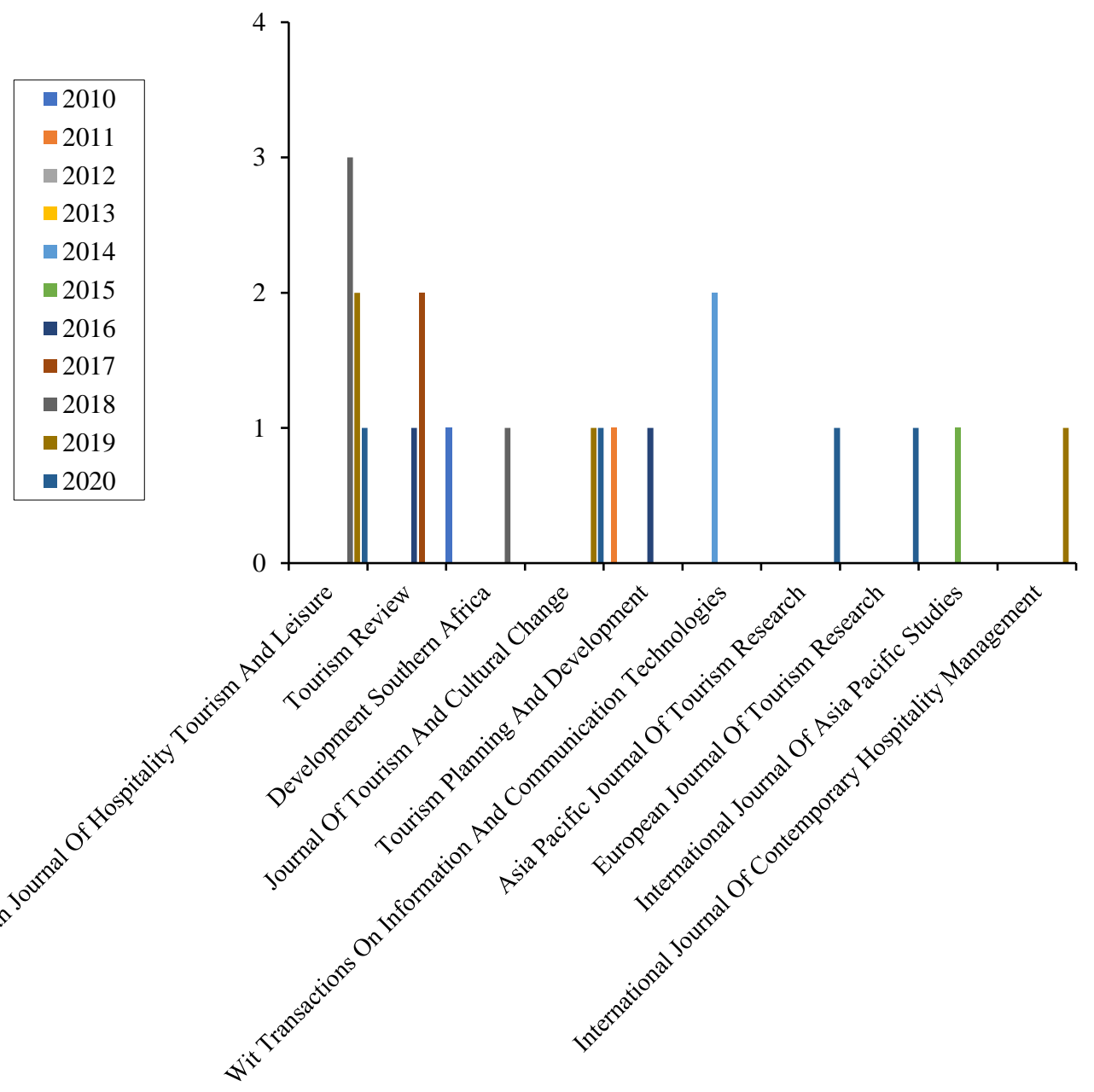

Figura 8. Documentos publicados por año y por revista. Elaboración con datos de www.scopus.com.

\section{Conclusiones}

Como se pudo apreciar a lo largo de este documento, los estudios sobre cadenas de valor turísticas de los últimos diez años son pocas, sin embargo, pueden sentar las bases de este tema y visibilizar la importancia de la investigación de cadenas de valor turísticas para lograr un desarrollo equitativo. Al respecto se tiene que los años más productivos con relación al tema fueron 2019 y 2020 con siete y nueve documentos respectivamente. Entre los primeros cuatro autores se encuentran Adiyia, Casarin, Halpern y Kaewkitipong. En lo que respecta a la afiliación, en primer lugar, se encuentra la Universidad de Innsbruck, una de las más antiguas de Austria. 
De acuerdo con el Ranking de Turismo Internacional (OMT, 2018), Tailandia ocupó el 4º lugar por ingreso de divisas de turismo y el $9^{\circ}$ por llegada de turistas, lo que podría explicar su interés en la investigación de cadenas de valor turísticas ya que es el país líder en investigaciones con esta temática. Cabe mencionar que América Latina tiene poca presencia en investigaciones sobre CVT, sin embargo, se reconocen las aportaciones científicas que han realizado Argentina, Colombia y Ecuador, mismas que podrían ser una base para México.

Predomina la modalidad de artículos científicos sobre la escritura de capítulos de libros y otros escritos, además de ser el área de negocios, gestión y contabilidad la más interesada en escribir sobre CVT. Lo anterior, puede explicarse ya que al turismo siempre se le ha visto como una actividad netamente económica; sin embargo, otras ciencias como las sociales y las mediambientales buscan visibilizar el lado humano del turismo y su importancia para lograr un desarrollo sustentable y equitativo para las comunidades. Finalmente, la revista African Journal Of Hospitality, Tourism and Leisure es la más popular entre los investigadores para publicar sus documentos. Presenta un CiteScore de 1.1 que es el promedio de citas recibidas por documento publicado en la serie (Scopus, 2020).

\section{Literatura citada}

Adiyia, B. and Vanneste, D. 2018. Local tourism value chain linkages as pro-poor tools for regional development in Western Uganda. Development Southern Africa. 35(2):210-224.

Adiyia, B.; Stoffelen, A.; Jennes, B.; Vanneste, D. and Ahebwa, W. 2015. Analysing governance in tourism value chains to reshape the tourist bubble in developing countries: the case of cultural tourism in Uganda. J. Ecotourism. 14(2-3):113-129.

Alonso, G. 2008. Marketing de servicios: reinterpretando la cadena de valor. Rev. Management de la Universidad de Palermo. Edición No. 2. Argentina. 83-96 pp.

Anderson, W. 2018. Linkages between tourism and agriculture for inclusive development in tanzania: A value chain perspective. J. Hosp. Tour. Manag. Insights. 1(2):168-184.

Anderson, W.; Busagara, T.; Mahangila, D.; Minde, M.; Olomi, D. and Bahati, V. 2017. The dialogue and advocacy initiatives for reforming the business environment of the tourism and hospitality sector in tanzania. Tourism Review. 72(1):45-67.

Castillo, V.; Figal-Garone, L.; Maffioli, A. and Salazar, L. 2017. The causal effects of regional industrial policies on employment: A synthetic control approach. Regional Sci. Urban Econ. 67:25-41.

Duque, E. 2005. Revisión del concepto de calidad del servicio y sus modelos de medición. Innovar. Rev. Cienc. Admin. Social. Colombia. 25(15):64-80.

FAO. 2006. Organización de las Naciones Unidas para la Alimentación y la Agricultura. Análisis prospectivo de política para la integración de cadenas. http://www.sagarpa.gob.mx /programas/evaluacionesExternas/Lists/Otros\%20Estudios/Attachments/10/Prospectivo\% 20Cadenas.pdf. 55 p.

GTZ. 2007. Agencia Alemana de cooperación técnica. Manual valuelinks metodología para el fomento de la cadena de valor. Alemania. $253 \mathrm{p}$.

González, J. 2015. La cadena de valor del servicio. https://es.linkedin.com/pulse/la-cadena-devalor-del-servicio-juan-manuel-gonz\%C3\%A1lez-s\%C3\%A1nchez. 
González, L. and Husain, S. 2020. Social entrepreneurship and sustainable tourism in Colombia: A baseline study in post-conflict regions. International Journal of sustainability in economic. Social, and Cultural Context. 16(2):65-85.

Imai, M. 1998. Cómo implementar el kaizen en el sitio de trabajo. McGraw-Hill Interamericana. Bogotá. 346 p.

Izaguirre, M. 2015. Gestión y marketing de servicios turísticos y hoteleros. ECOE Ediciones. $2^{\mathrm{a}}$ (Ed.). Bogotá, Colombia. 182 p.

Kaewkitipong, L. 2010a. Disintermediation in the tourism industry: theory vs practice. In: Nelson, M., Shaw, M., \& Strader T. (eds) Sustainable e-Business Management. AMCIS 2010. Lecture notes in business information processing. 58:160-170.

Kaewkitipong, L. 2010b. Disintermediation in the tourism industry: theory vs. practice. $16^{\text {th }}$ America's conference on information systems. 3:2277-2285.

Kaplinsky, R. and Morris, M. 2001. A Handbook for value chain research. Brighton, United Kingdom-Institute of Development Studies. University of Sussex. 114 p.

Kaplinsky, R. 2000. Globalisation and unequalisation: what can be learned from value chain analysis? J. Develop. Studies. 2(37):117-146.

Kippenberger, T. 1997. The value chain: the original breakthrough. The Antidote. 5(2):7-10.

Kotler, P. 1997. Mercadotecnia. Prentice-Hall. México, DF. 656 p.

Kotler, P.; Bowen, J.; Makens, J.; García, J. y Flores, J. 2011. Marketing turístico. $5^{\text {ta }}$ (Ed.). Pearson. Madrid. 827 p.

Manente, M.; Minghetti, V.; Mingotto, E. and Casarin, F. 2014. Consumer confidence in responsible tourism: How business reporting systems can encourage respectful behaviour. Handbook of research on consumerism in business and marketing: concepts and practices. 264-285 p.

Manente, M.; Minghetti, V.; Mingotto, E. and Casarin, F. 2014. Consumer confidence in responsible tourism: how business reporting systems can encourage respectful behaviour. Hospitality, Travel, and Tourism: Concepts, Methodologies, Tools, and Applications. 3:1406-1427.

Mwesiumo, D. and Halpern, N. 2018. Acquiescence and conflict in exchanges between inbound tour operators and their overseas outbound partners: a case study on Tanzania. Tourism Management. 69:345-355.

Mwesiumo, D. and Halpern, N. 2016. Interfirm conflicts in tourism value chains. Tourism Rev. 71(4):259-271.

Observatorio laboral. 2020. Información estadística para el futuro académico y laboral en México. Primer trimestre. Ocupación por sectores económicos. https://www.observatoriolaboral.gob.mx/static/estudios-publicaciones/Ocupacion_ sectores.html.

OMT. 2015. Organización Mundial del Turismo. Panorama OMT del turismo internacional. 16 p.

OMT. 2018. Organización Mundial del Turismo. Barómetro de la OMT. Ranking mundial del turismo internacional. https://www.datatur.sectur.gob.mx/SitePages/RankingOMT.aspx.

Ortiz, G.; Branco, A.; Sancho, P. and Castillo, J. 2001. ESTIA-efficient electronic services for tourists in action. Lecture Notes in Computer Science. 2193:163-174.

Porter, M. and Millar, V. 1985. How information gives you competitive advantage. Harvard Bussiness Review. 14 p. 
Pulido, J. y López, Y. 2016. La cadena de valor del destino como herramienta innovadora para el análisis de la sostenibilidad de las políticas turísticas. El caso de España. Innovar. Rev. Cienc. Admin. Soc. 59(26):155-175.

Rendón, M. and Bidwell, S. 2015. Success in progress? Tourism as a tool for inclusive development in Peru's Colca Valley. Tourism in Latin America: cases of success. 207-213 pp.

Robert, L.; Richard, C. and Harsha, C. 1989. Marketing leadership in hospitality. Foundations and Practices. 844 p.

Romero, I. and Tejada, P. 2011. A multi-level approach to the study of production chains in the tourism sector. Tourism Management. 32(2):297-306.

Scopus. 2020. Detalle de fuente. Afr. J. Hospitality, Tourism Leisure. https://www.scopus.com/ sourceid/21100829917? origin=resultsAnalyzer\&zone=sourceTitle.

Serrano, J. 2011. El sector servicios en la economía global: transformaciones y consecuencias. Clío. History and history teaching No. 37.17 p. http://clio.rediris.es/n37/oposiciones $2 /$ tema08.pdf.

Uduji, J.; Okolo-Obasi, E. and Asongu, S. 2019. Does CSR contribute to the development of rural young people in cultural tourism of Sub-Saharan Africa? Evidence from the Niger Delta in Nigeria. J. Tourism Cultural Change. 17(6):725-757.

Uduji, J.; Okolo-Obasi, E.; Onodugo, V.; Nnabuko, J. and Adedibu, B. 2020. Corporate social responsibility and the role of rural women in strengthening agriculture-tourism linkages in nigeria's oil producing communities. J. Tourism Cultural Change. 38 p.

Zeithaml, A.; Bitner, J. y Gremler, D. 2009. Marketing de servicios. McGraw-Hill Interamericana de España, SL. $5^{\text {a }(E d .) . ~} 740$ p. 